\title{
RIFLE-Based Data Collection/Management System Applied to a Prospective Cohort Multicenter Italian Study on the Epidemiology of Acute Kidney Injury in the Intensive Care Unit
}

\author{
Francesco Garzotto $^{b, c}$ Pasquale Piccinni ${ }^{a}$ Dinna Cruz ${ }^{\mathrm{b}, c}$ Silvia Gramaticopolo ${ }^{\mathrm{a}}$ \\ Marzia Dal Santo ${ }^{a}$ Giovanni Aneloni ${ }^{c}$ Jeong Chul Kim ${ }^{c}$ Monica Rocco ${ }^{d}$ Elisa Alessandrid \\ Francesco Giunta ${ }^{f}$ Vincenzo Michetti ${ }^{\mathrm{e}}$ Michele lannuzzi ${ }^{g}$ Clara Belluomo Anello ${ }^{\text {h }}$ \\ Nicola Brienza ${ }^{i}$ Mauro Carlini ${ }^{j}$ Paolo Pelaiak ${ }^{k}$ Vincenzo Gabbanellik Claudio Ronco ${ }^{b, c}$ \\ on behalf of the NEFROINT investigation group \\ Departments of antensive Care and Anesthesiology, and ${ }^{\mathrm{b}}$ Nephrology, Dialysis and Transplantation, St. Bortolo \\ Hospital, and 'International Renal Research Institute Vicenza, Vicenza, ${ }^{\mathrm{d}}$ Department of Anesthesiology and \\ Intensive Care, University of Rome 'La Sapienza', e'Department of Intensive Care and Anesthesiology, Catholic

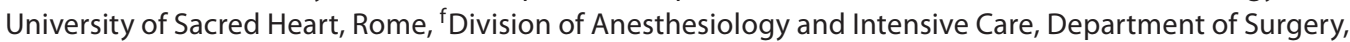 \\ University of Pisa, Pisa, 9 Intensive Care Unit, Department of Anesthesia and Resuscitation, Federico II University \\ Hospital, Naples, hDepartment of Anesthesia and Intensive Care, San Giovanni di Dio Hospital, Florence, \\ 'Department of Anesthesia and Intensive Care, Emergency and Organ Transplantation, University of Bari, Bari,

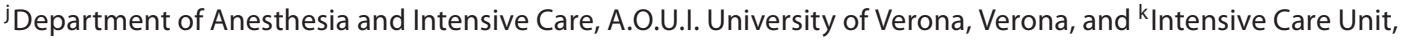 \\ University Hospital Umberto I, G.M. Lancisi, G. Salesi, Ancona, Italy
}

\section{Key Words}

Acute kidney injury, epidemiology • Acute renal failure • Intensive care unit $\cdot$ Renal replacement therapy $\cdot$ Sepsis

\begin{abstract}
The epidemiology of acute kidney injury (AKI) has been difficult to explore in the past, due to different definitions across various studies. Nevertheless, this is a very important topic today in light of the high morbidity and mortality of critically ill patients presenting renal dysfunction during their stay in the intensive care unit (ICU). The case mix has changed over the years, and AKI is a common problem in critically ill patients often requiring renal replacement therapy (RRT). The RIFLE and AKIN initiatives have provided a unifying definition for AKI, making possible large retrospective studies in different countries. The present study aims at vali-
\end{abstract}

dating a unified web-based data collection and data management tool based on the most recent AKI definition/classification system. The interactive database is designed to elucidate the epidemiology of AKI in a critically ill population. As a test, we performed a prospective observational multicenter study designed to prospectively evaluate all incident admissions in ten ICUs in Italy and the relevant epidemiology of AKI. Thus, a simple user-friendly web-based data collection tool was created with the scope to serve for this study and to facilitate future multicenter collaborative efforts. We enrolled 601 consecutive incident patients into the study; 25 patients with end-stage renal disease were excluded, leaving 576 patients for analysis. The median age was 66 (IQR 53-76) years, 59.4\% were male, while median Simplified Acute Physiology Score II and Acute Physiology and Chronic Health Evaluation II scores were 43 (IQR 35-54) and 18 (IQR 13-24), respectively. The most common diagnostic catego-

\section{KARGER}

Fax +41613061234 E-Mail karger@karger.ch www.karger.com
(C) 2011 S. Karger AG, Basel

0253-5068/11/0313-0159\$38.00/0

Accessible online at:

www.karger.com/bpu
Prof. Claudio Ronco, Editor-in-Chief

Department of Nephrology, Dialysis and Transplantation

San Bortolo Hospital, Viale Rodolfi 37

IT-36100 Vicenta (Italy)

Tel. +39 0444753 869, Fax +390444 753 939, E-Mail cronco@goldnet.it 
ries for ICU admission were: respiratory (27.4\%), followed by neurologic (17\%), trauma (14.4\%), and cardiovascular (12.1\%). Crude ICU and hospital mortality were $21.7 \%$ and median ICU length of stay was 5 (IQR 3-14) days. Of 576 patients, 246 patients (42.7\%) had AKI within $24 \mathrm{~h}$ of ICU admission, while 133 developed new AKI later during their ICU stay. RIFLE-initial class was Risk in 205 patients (54.1\%), Injury in 99 (26.1\%) and Failure in 75 (19.8\%). Progression of AKI to a worse RIFLE class was seen in 114 patients (30.8\% of AKI patients). AKI patients were older, with higher frequency of common risk factors. $116 \mathrm{AKI}$ patients (30.6\%) fulfilled criteria for sepsis during their ICU stay, compared to $33(16.7 \%)$ of non-AKI patients $(p<0.001) .48$ patients $(8.3 \%)$ were treated with RRT in the ICU. Patients were started on RRT a median of 2 (IQR 0-6) days after ICU admission. AKI patients were started on RRT a median of 1 (IQR 0-4) day after fulfilling criteria for AKI. Median duration of RRT was 5 (IQR 2-10) days. AKI patients had a higher crude ICU mortality (28.8 vs. $8.1 \%$, non-AKI; $\mathrm{p}<$ 0.001) and longer ICU length of stay (median 7 vs. 3 days, non-AKI; $\mathrm{p}<0.001)$. Crude ICU mortality and ICU length of stay increased with greater severity of AKI. 225 (59.4\% of AKI patients) had complete recovery of renal function, with a serum creatinine at time of ICU discharge which was $\leq 120 \%$ of baseline; an additional 51 AKI patients (13.5\%) had partial renal recovery, while 103 (27.2\%) had not recovered renal function at the time of death or ICU discharge. The study supports the use of RIFLE as an optimal classification system to stage AKI severity. AKI is indeed a deadly complication for ICU patients, where the level of severity is correlated with mortality and length of stay. The tool developed for data collection was user-friendly and easy to implement. Some of its features, including a RIFLE class alert system, may help the treating physician to systematically collect AKI data in the ICU and possibly may guide specific decisions on the institution of RRT.

Copyright $\odot 2011$ S. Karger AG, Base

\section{Introduction}

The epidemiology of acute kidney injury (AKI) has been difficult to explore in the past due to different definitions across various studies. Nevertheless, this is a very important topic today in light of the high morbidity and mortality of critically ill patients presenting renal dysfunction during their stay in the intensive care unit (ICU) [1]. The case mix has changed over the years, and AKI is a common problem in critically ill patients often requiring renal replacement therapy (RRT). The RIFLE and AKIN initiatives have provided a unifying definition for
AKI, making possible large retrospective studies in different countries [2-5]. RIFLE/AKIN define and stage AKI using serum creatinine (SCr), urine output (UO) and need for RRT, making them simple to apply in a variety of clinical and research settings [3]. The use of such definitions in the literature has increased gradually over the last 6 years [4-11]. The present study aims at validating a unified web-based data collection and data management tool based on the most recent AKI definition/classification system. A simple user-friendly web-based data collection tool was created with the scope to serve for this study and to facilitate future multicenter collaborative efforts. The interactive database is designed to elucidate the epidemiology of AKI in a critically ill population. As a test, we performed a prospective observational multicenter study designed to prospectively evaluate all incident admissions in ten ICUs in Italy and the relevant epidemiology of AKI. Our objectives were to determine the incidence of AKI among critically ill patients using consensus definitions, to characterize etiology and timing of AKI, and to evaluate clinical outcomes associated with AKI.

\section{Methods}

Creation of Database and Test Application

Although the evolution of numerous tools and methods has greatly improved over the years, the process of collecting accurate data can still be difficult; this is particularly true in situations where there are multiple study sites or offsite data collection is involved. The achievement of a well-established methodology often results from a multiple steps process that involves multidisciplinary fields like medical, engineering and statistical. Hence, in order to create a user-friendly data collection system, applicable in several ICUs, we undertook tree subsequent development steps.

The archaic version of the electronic case report form was developed, as an Excel file, to evaluate the quality of the contribution of every field to the statistical analysis and the major error committed filling in the data (accuracy and compliance). A second system based on HTML and MS Access database permitted to eliminate the previous critical issues and to increment the cooperation of different centers allowing multiple simultaneous access from different locations; an evaluation of time spent to manage the information required was moreover estimated to improve next version. 300 patients in this step were enrolled as a pilot study. The third final and current generation of the program was based on the latest open source framework functionality. As a test of this latest version, a prospective observational study was done on adult patients (age $\geq 18$ years) admitted to ten ICUs in Italy from September 2009 to April 2010 (participating centers are listed at the end of the article). The study protocol was reviewed by the ethics committees or institutional review boards of the participating centers. Because of the anonymous and noninterventional nature of the study, the ethics committees of study centers waived the need for informed consent. 
AKI was defined using both the creatinine and UO criteria of the RIFLE classification [1]. A patient was considered to have AKI when he had an increase in SCr of at least $50 \%$ from baseline, or a reduction in $\mathrm{UO}$ to $<0.5 \mathrm{ml} / \mathrm{kg} / \mathrm{h}$ for more than $6 \mathrm{~h}$ (i.e. fulfilling at least class Risk). Clinical charts were reviewed for previous SCr values. Baseline renal function was defined as the lowest known SCr value during the preceding 3 months. For patients without known prior SCr, the baseline SCr was estimated by solving the MDRD equation, assuming a glomerular filtration rate of $75 \mathrm{ml} / \mathrm{min} / 1.73 \mathrm{~m}^{2}$, as recommended by the ADQI Working Group [1] and previously applied $[10,12]$. Patients were classified daily using the RIFLE criteria. RIFLE-initial refers to their RIFLE class on the first day of AKI. RIFLE-max refers to the worst RIFLE class reached by a patient during his ICU stay. Patients were considered as having new AKI if they did not have AKI on ICU admission, but subsequently reached at least class Risk during their follow-up. AKI patients were considered as having worsening AKI if they later reached a higher RIFLE-max class compared to RIFLE-initial (i.e. Risk to Injury or Failure, or Injury to Failure) at any time during their ICU stay. Patients with RIFLE-initial class of Failure were considered as having worsening AKI if they later required RRT. RRT was initiated and conducted at the discretion of the responsible physicians. There were no standardized criteria to start or end RRT. For AKI patients, renal outcome at ICU discharge or death was defined as follows: complete recovery if discharge SCr was within $120 \%$ of baseline; partial recovery if discharge SCr was 121-150\% of baseline, and non-recovery if discharge SCr was $>150 \%$ of baseline or still receiving RRT.

Sepsis and systemic inflammatory response syndrome were diagnosed according to the definitions of the American College of Chest Physicians/Society of Critical Care Medicine [13]. Patients who fulfilled these criteria during their ICU admission were classified accordingly. The individual data elements for the Acute Physiology and Chronic Health Evaluation (APACHE) II score [14], Sequential Organ Failure Assessment (SOFA) score [15] and Simplified Acute Physiology Score (SAPS) II [16] were recorded on the day of ICU admission, and scores were automatically calculated. SOFA score was calculated daily.

\section{Data Collection}

Data from enrolled patients were entered into electronic case report forms resident on a password-protected web application. Centers could only have access to data relevant to their patients. Multiple data elements were collected for each patient. After usability test, a web-based system was designed based on an open source framework Ruby on Rails. Data were collected into a Mysql database. A web-based system was chosen to offer investigators the convenience of collecting and entering information from various locations within their own centers in a rapid, efficient and accurate manner. The web further offers the advantages of both centralization of information and coordination of multiple clinical trial processes. An automatic data verification system for each data field screens for missing or out of range values and data inconsistencies, and generates a visual user alert. The user is informed about the error and is asked to correct it in real time. The web tool is organized in eight distinct sections, with easy navigability from one section to another: (1) Demographics, anthropometrics, and admission diagnoses; (2) Comorbidities; (3) First ICU day; (4) Daily vital signs and laboratory values; (5) AKI; (6) RRT; (7) Sepsis, and (8) Outcomes/Case closure. In the 'Comorbidities' section, a his- tory of exposure to nonsteroidal anti-inflammatory drugs, angiotensin-converting enzyme inhibitors (ACE-I), angiotensin-2-receptor blockers (ARB), aminoglycosides or radiocontrast prior to ICU admission was also recorded. Data from the 'First ICU day' section are used to automatically calculate APACHE II, SAPS II and SOFA scores. In the 'Daily vital signs and laboratory values' section, the 24-hour UO, the worst 12-hour UO, the worst 6-hour $\mathrm{UO}$, and the worst SCr for that day are entered. The system then automatically displays the following items: the ratio of the day's SCr to the baseline SCr, the ratio of the day's SCr to the SCr within the previous 48-hour window, and the UO expressed as $\mathrm{ml} /$ $\mathrm{kg} / \mathrm{h}$. These aid the investigator in selecting the appropriate RIFLE and AKIN stage in the 'AKI' section. An optional alert can be activated when the patient reaches at least RIFLE class Risk. Use of diuretics was also recorded. In the 'AKI' section, the suspected factors contributing to AKI are entered. The 'RRT' section records indication for RRT, details of RRT prescription and delivery. In the 'Sepsis' section, the microorganism(s) and suspected source of infection are recorded. For 'Outcome', all-cause ICU and hospital (when available) mortality are entered. Periodic audits were performed to establish the accuracy of data capture and transfer into the database. An e-mail and/or telephone contact was made to the participating investigators requesting completion, correction, or verification of specific data items. Edit messages are retained in the file indicating the questionable data and any changes made when the requested information is returned. The final data were then imported into a statistical program for analysis.

Continuous variables are expressed as mean \pm standard deviation or median (interquartile range) and compared between any two groups using $t$ test or the Mann-Whitney $U$ test, and among three groups using analysis of variance (general linear models with adjustment for multiple comparisons), as appropriate. Categorical variables are expressed as proportions and compared with the Mantel-Haenszel $\chi^{2}$ test or Fisher exact test. Data were analyzed using the SPSS 14.0 (SPSS, Inc., Chicago, Ill., USA) software package, with two-sided $p$ value $<0.05$ considered as statistically significant.

At the end of the study, a cross-check with the study participants and the internal review committee was done in order to establish a final evaluation of the data collection tool. The interface was evaluated in terms of user friendliness; the access to data input and data retrieval was discussed, and the easy transfer to a statistical program was also evaluated. A final general conclusion on scalability and applicability in future studies was then drawn from the obtained evaluations and results.

\section{Data Collection Tool and Database Evaluation Results}

The final version of the database collection tool has been defined by the users as easy to implement in the clinical routine. This indeed may become an important tool to collect patient's information, build statistics and generate epidemiology trials.

In figure 1 , a screenshot of the patient management system is depicted. Figure 2 describes a step for a 'new 


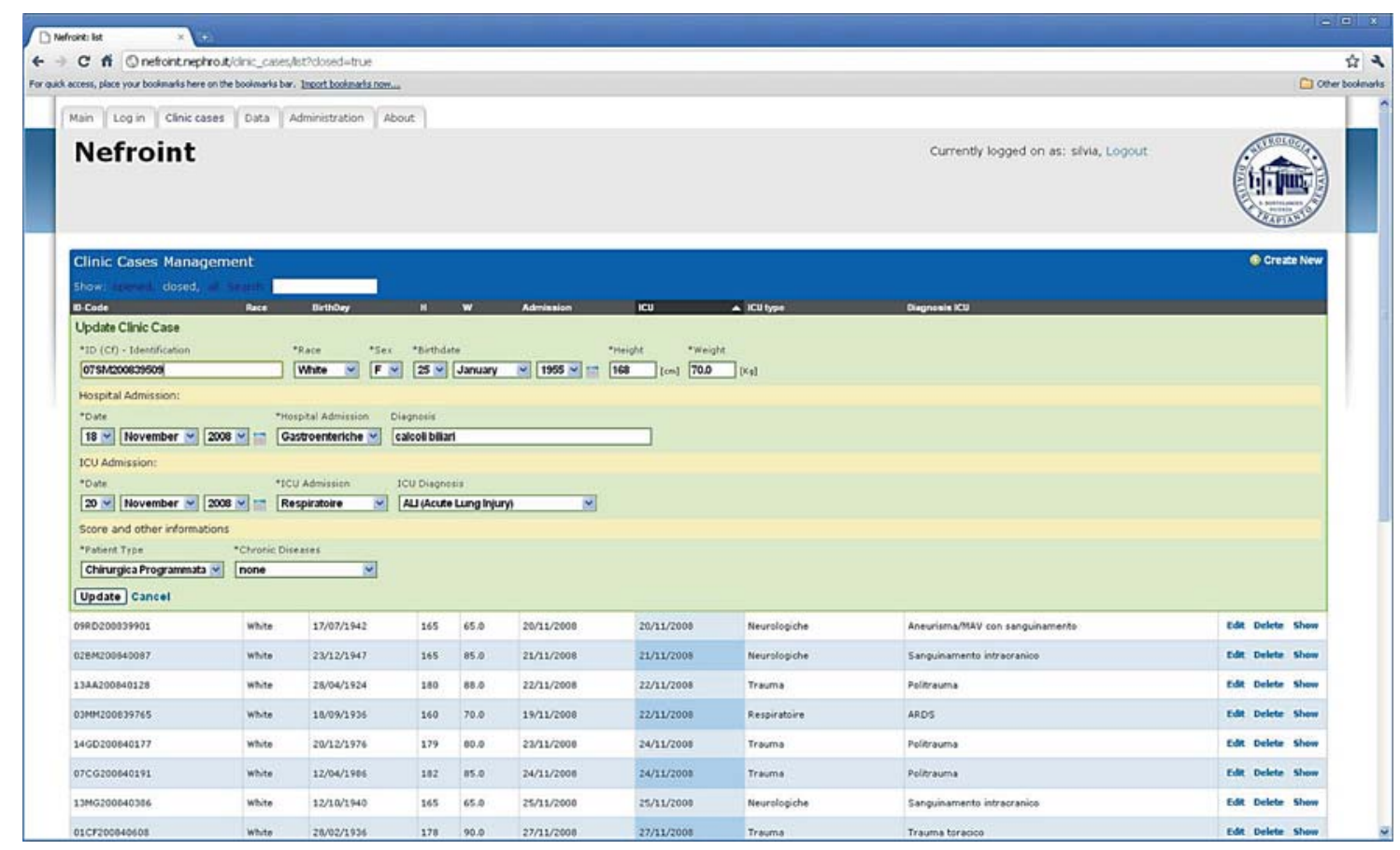

Fig. 1. Patient management. A screen shot of the first page of the NEFROINT system. The list of the patients recorded displays the most important information to be inserted. Edit and Delete fields are available on the right. With the Show button, one may enter the specific Patient CRF.

patient' creation. At any time, review of data recorded is possible as shown in figures 3 and 4 . The MDRD calculation of GFR is useful to estimate baseline creatinine and to help with RIFLE class definition.

In figures 5 and 6 , the most important laboratory data are displayed together with the fields required to calculate APACHE II, SAPS II and SOFA scores. New Lab records can be created with a simple but effective automatic data verification system (fig. 7, 8). An interesting feature of the program is the RIFLE alert alarm. When a patient reaches a given class of AKI, a pop-up alarm blinks, and the operator may decide for a RIFLE-based application of a RRT technique (fig. 9, 10). The database contains a section dedicated to continuous RRT (CRRT) (fig. 11) and a verification tool (fig. 12) to assess possible errors in data entry.

\section{Demographics and AKI Epidemiology Results}

We enrolled 601 consecutive incident patients into the study; we excluded 25 patients with end-stage renal disease on chronic RRT, leaving 576 patients for analysis.
Patient characteristics are shown in table 1 . The median age was 66 (IQR 53-76) years, 59.4\% were male, while median SAPS II and APACHE II scores were 43 (IQR 35-54) and 18 (IQR 13-24), respectively. The most common diagnostic categories for ICU admission were: respiratory (27.4\%), followed by neurologic (17\%), trauma (14.4\%), and cardiovascular (12.1\%). SCr values prior to hospitalization were available for only $45.8 \%$ of patients; for the rest, the baseline SCr was estimated using MDRD. The proportion of patients in which the MDRD estimation was used was similar among AKI and non-AKI patients. The median baseline premorbid SCr was 1.0 (IQR 0.8-1.1) $\mathrm{mg} / \mathrm{dl}$. Crude ICU and hospital mortality were $21.7 \%$, and median ICU length of stay was 5 (IQR 3-14) days.

Of 576 patients, 246 (42.7\%) had AKI within $24 \mathrm{~h}$ of ICU admission, while 133 developed new AKI later during their ICU stay (fig. 13). The most commonly reported factors contributing to AKI were hypovolemia (29.5\%), septic shock (13.5\%), major surgery (12.1\%) and cardiogenic shock (11.8\%). RIFLE-initial class was Risk in 205 patients (54.1\%), Injury in 99 (26.1\%) and Failure in 75 (19.8\%). Progression of AKI to a worse RIFLE class was seen in 114 patients (30.8\% of AKI patients). 


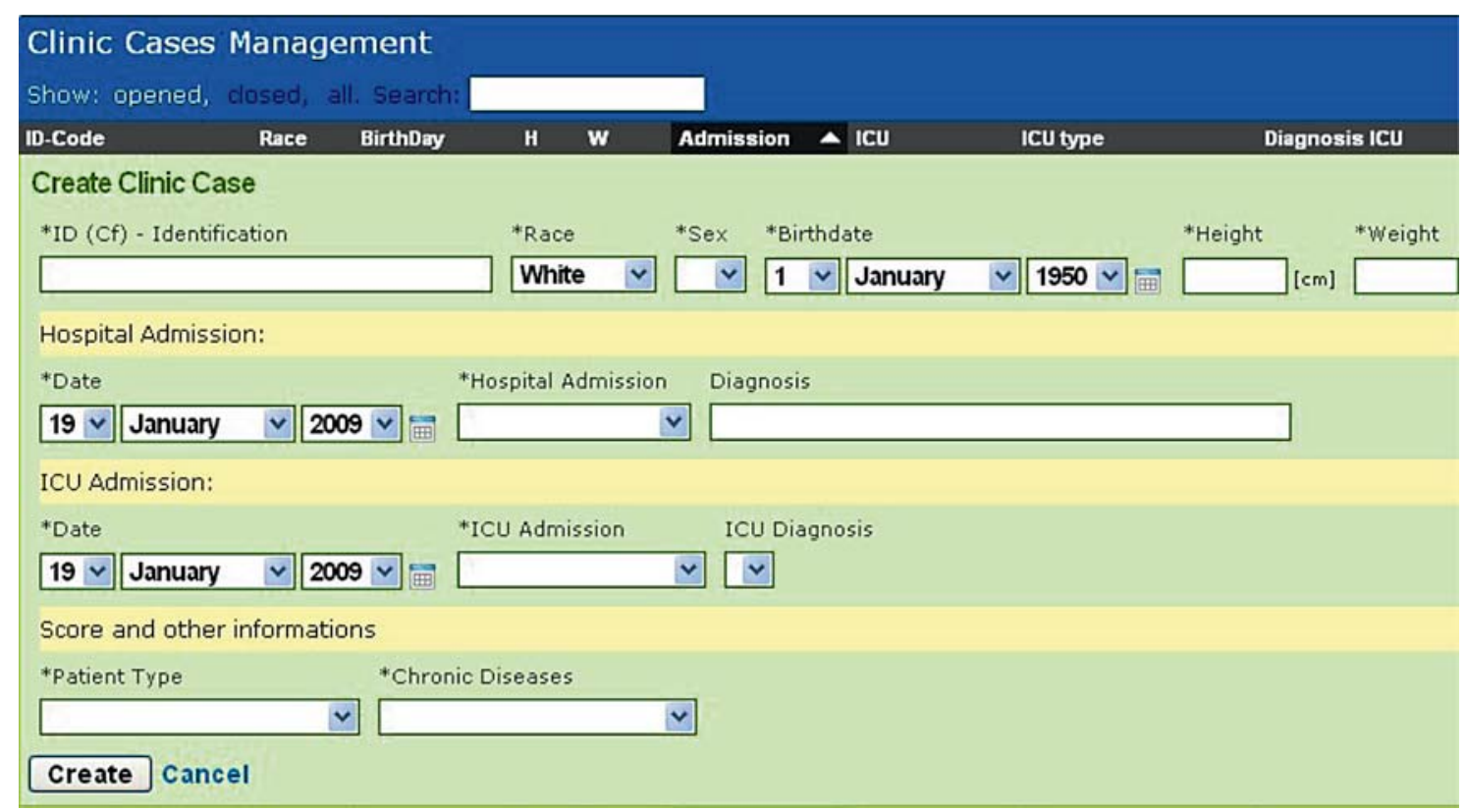

Fig. 2. Create new patient fields. Here, general patient information is collected to permit the identification of the patient type, the reason and the date of admission to hospital and ICU.

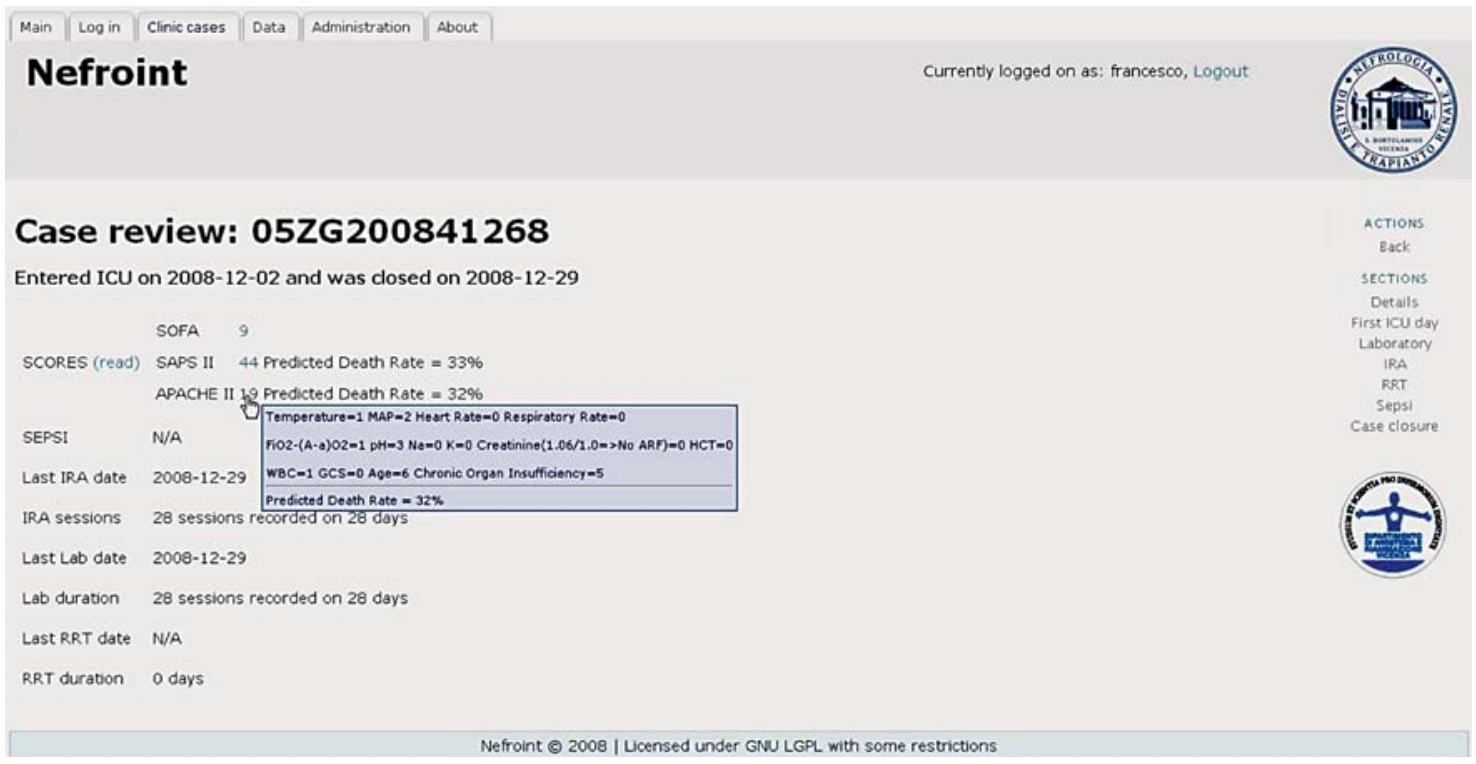

Fig. 3. Review page is useful to monitor the progress of the data recorded. The amount of days recorded and the last date for every section are reported. SOFA, SAPS II and APACHE II are automatically calculated from the 'First ICU day' section. Pop-up window reports the score details. 


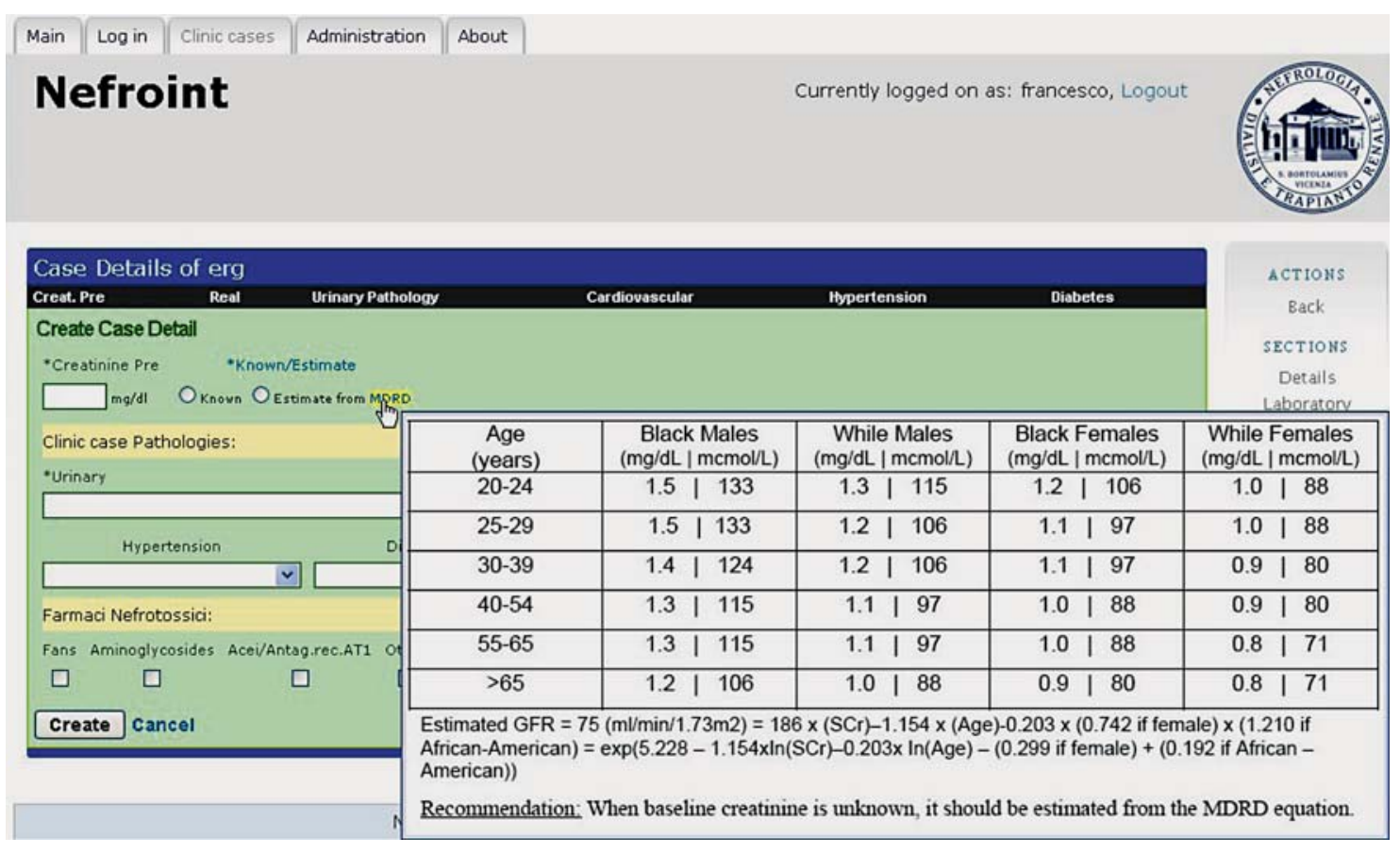

Fig. 4. Case Detail section. The figure shows the pop-up image that helps in MDRD estimation.

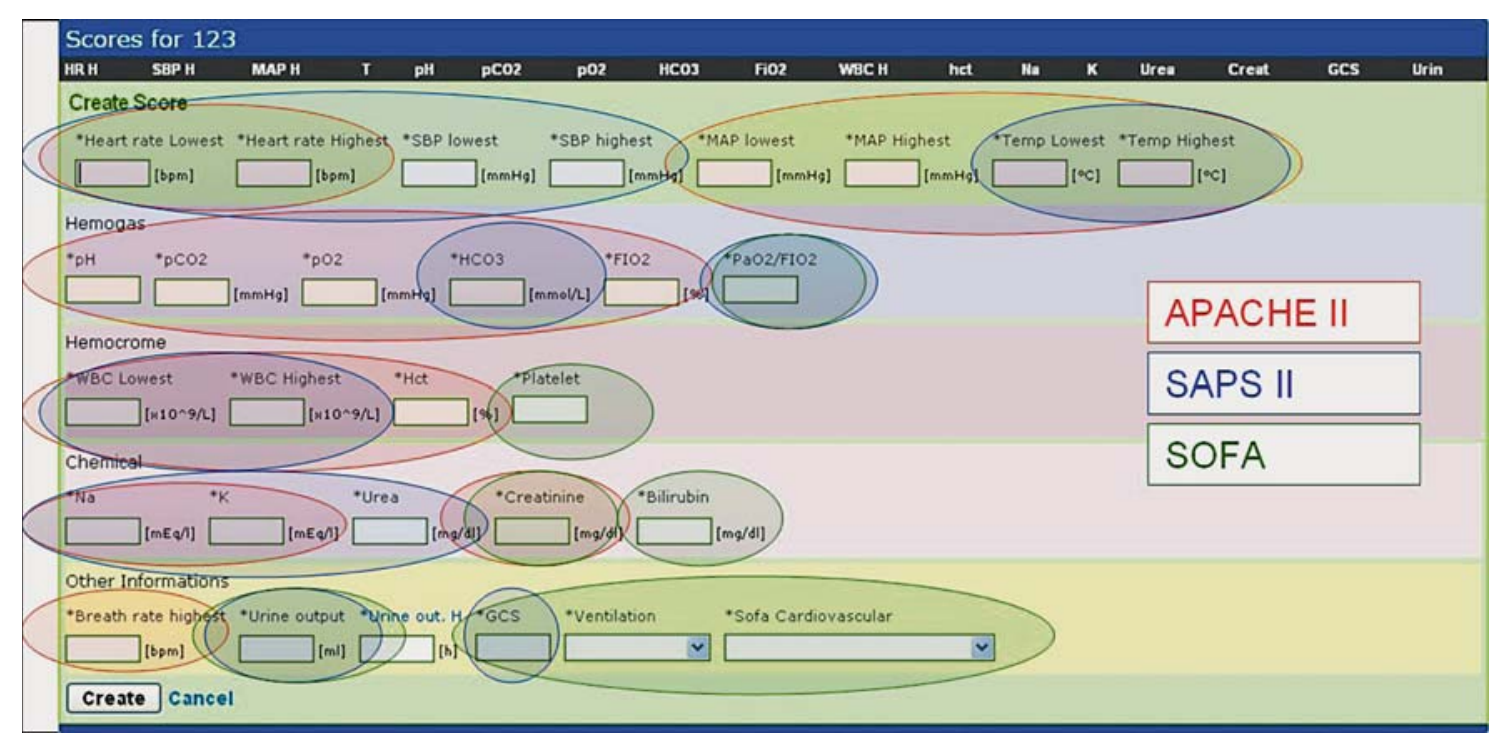

Fig. 5. First ICU day. Only 29 fields are required to calculate SOFA, SAPS II and APACHE II. Single score calculation would require the compilation of 39 fields: SOFA 8 fields, SAPS II 15 fields, APACHE II 16 fields; 39 in total. 


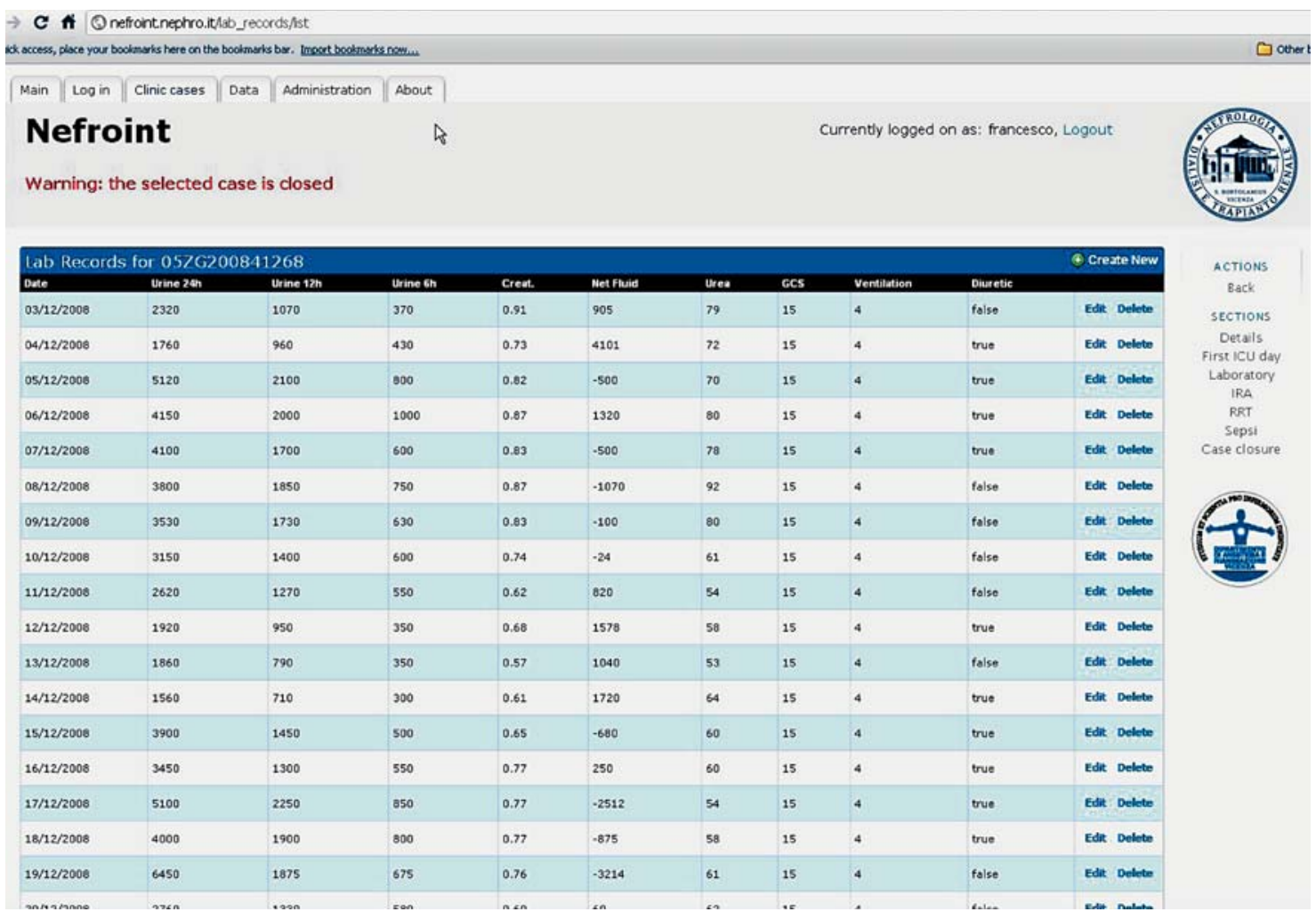

Fig. 6. The most important laboratory data are listed when the section is opened. Ascending and descending order is provided for date, UO, creatinine, net fluid balance, urea, etc. Edit and Delete functions on records are always available.

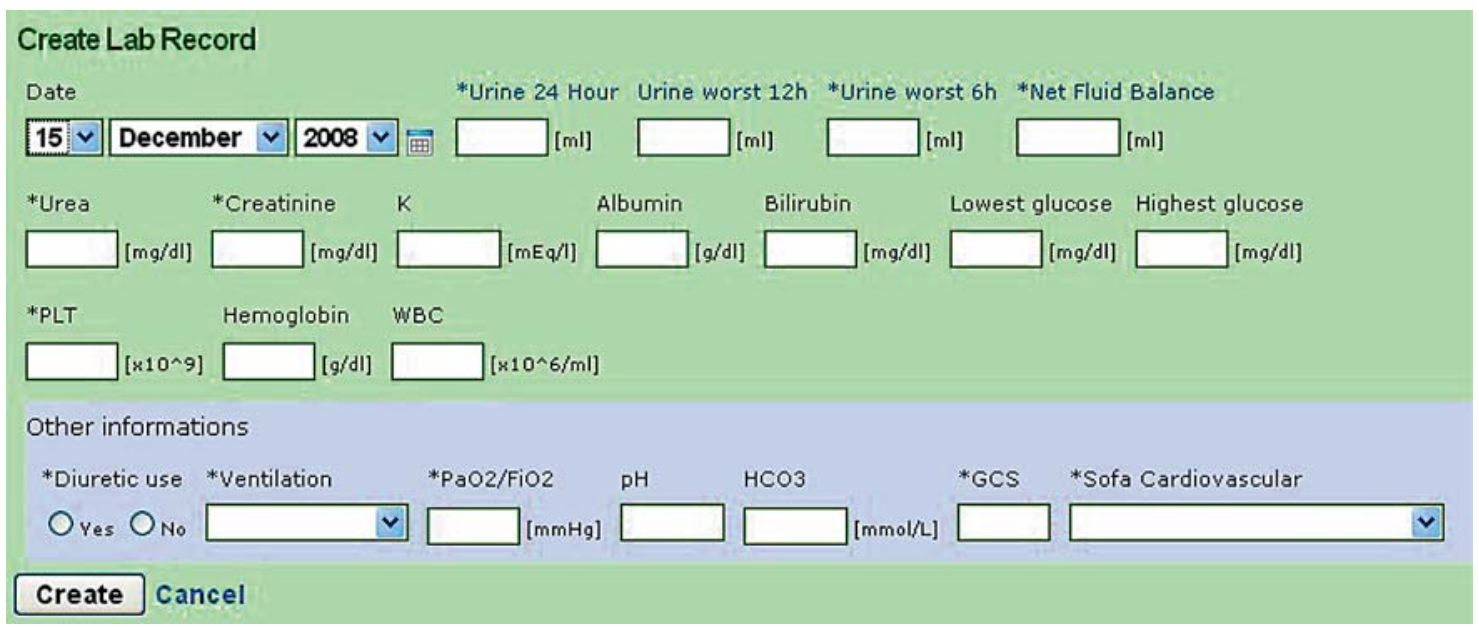

Fig. 7. The screen shot of 'Create Lab Record' displays twelve compulsory fields marked with an asterisk, as well as nine optional fields. Daily SOFA score is estimated by the 'Other informations' section. 


\section{"LABORATORY” SECTION}

Man, caucasic, 70Kg, Urin Output $6 \mathrm{~h}=190 \mathrm{ml}$

$190<0.5 * 70 * 6=210 \Rightarrow$ Urin Output $12 \mathrm{~h}$ is request for RIFLE class "I"

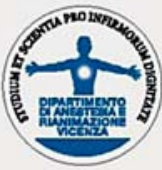

A

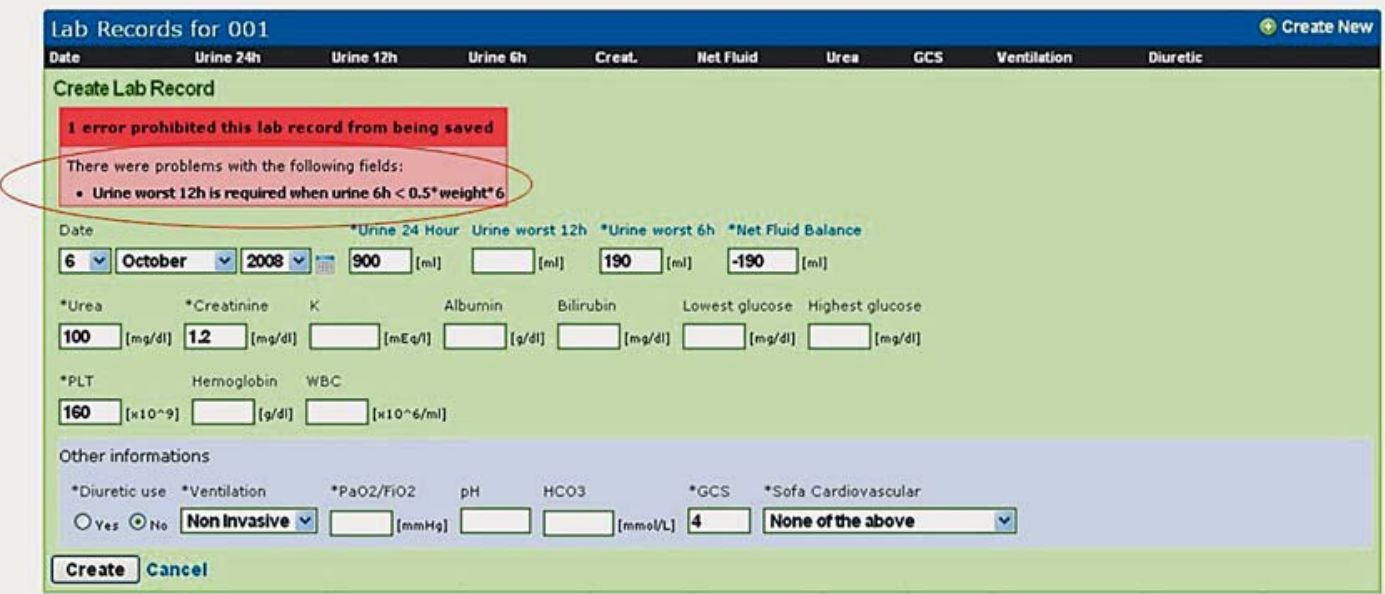

Fig. 8. Automatic data verification system for conditioned data. The displayed case requires 12 -hour UO to evaluate RIFLE I class.

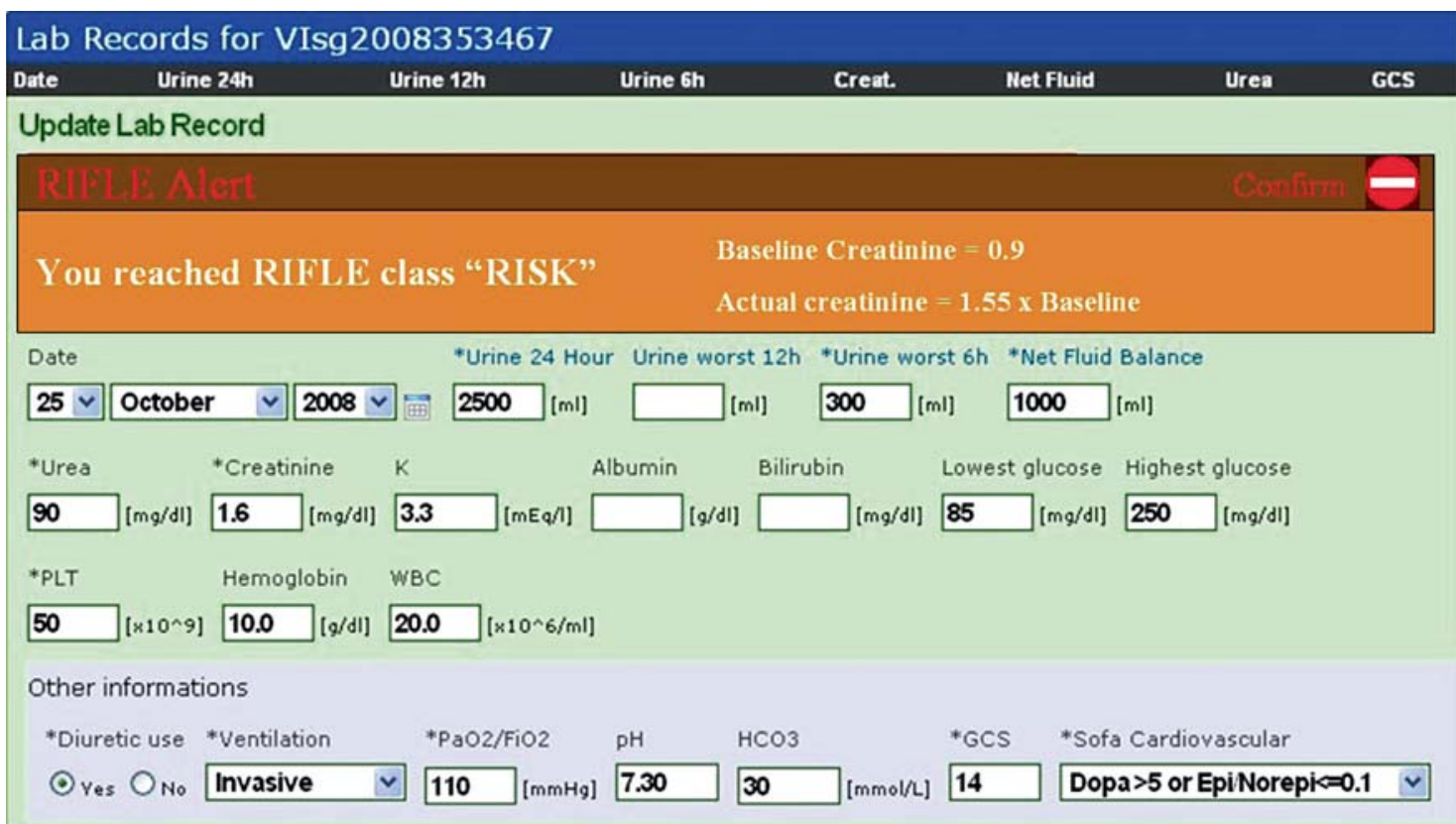

Fig. 9. A simple RIFLE alert can be activated to help in medical decision without any supplementary operation. Early start of CRRT may depend on this pop-up RIFLE class alarm. 


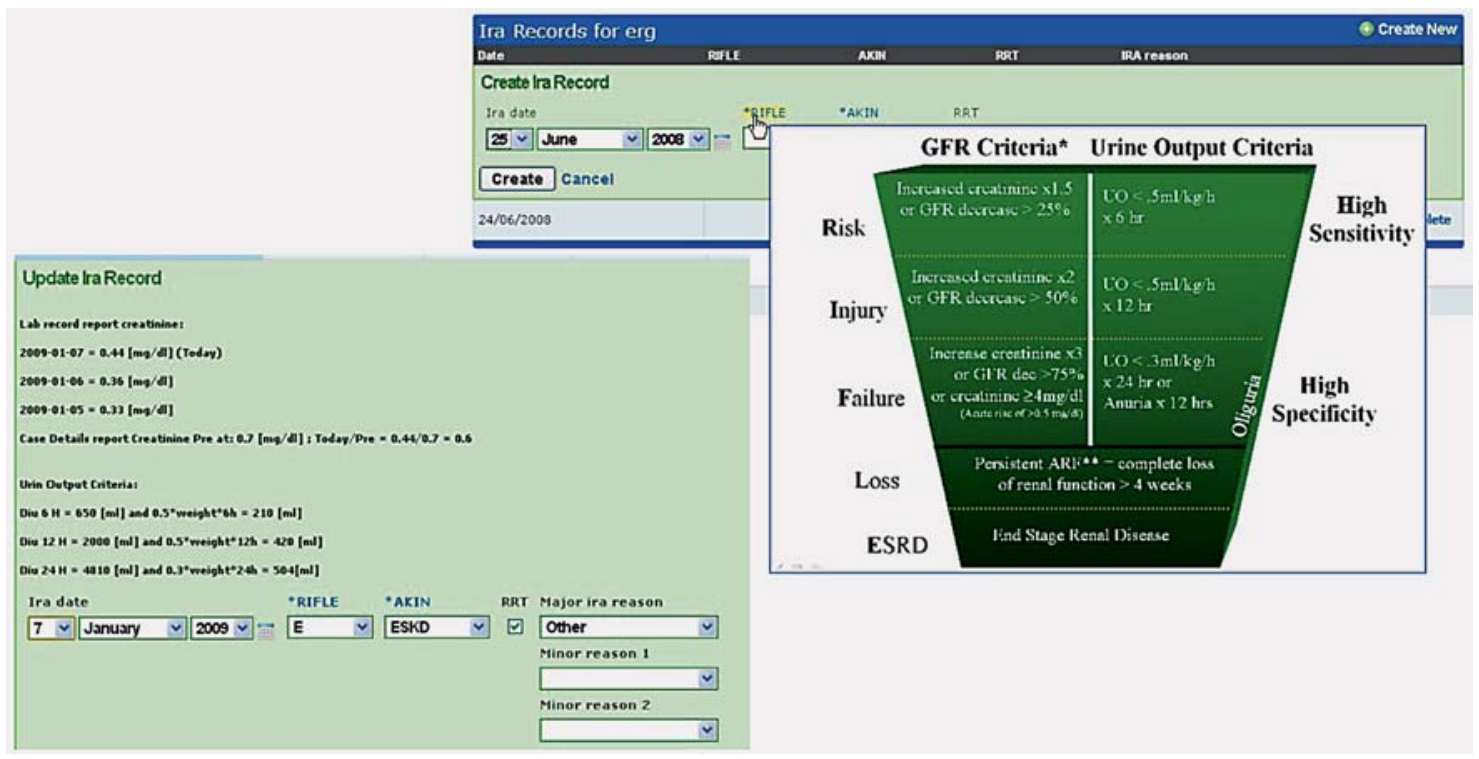

Fig. 10. RIFLE and AKIN criteria are supported by the corresponding decision schema. An important tool reports the ratio of the day's SCr to the baseline SCr, the ratio of the day's SCr to the SCr within the previous 48-hour window, and the UO expressed as $\mathrm{ml} / \mathrm{kg} / \mathrm{h}$. The criteria are estimated without any type of calculation.

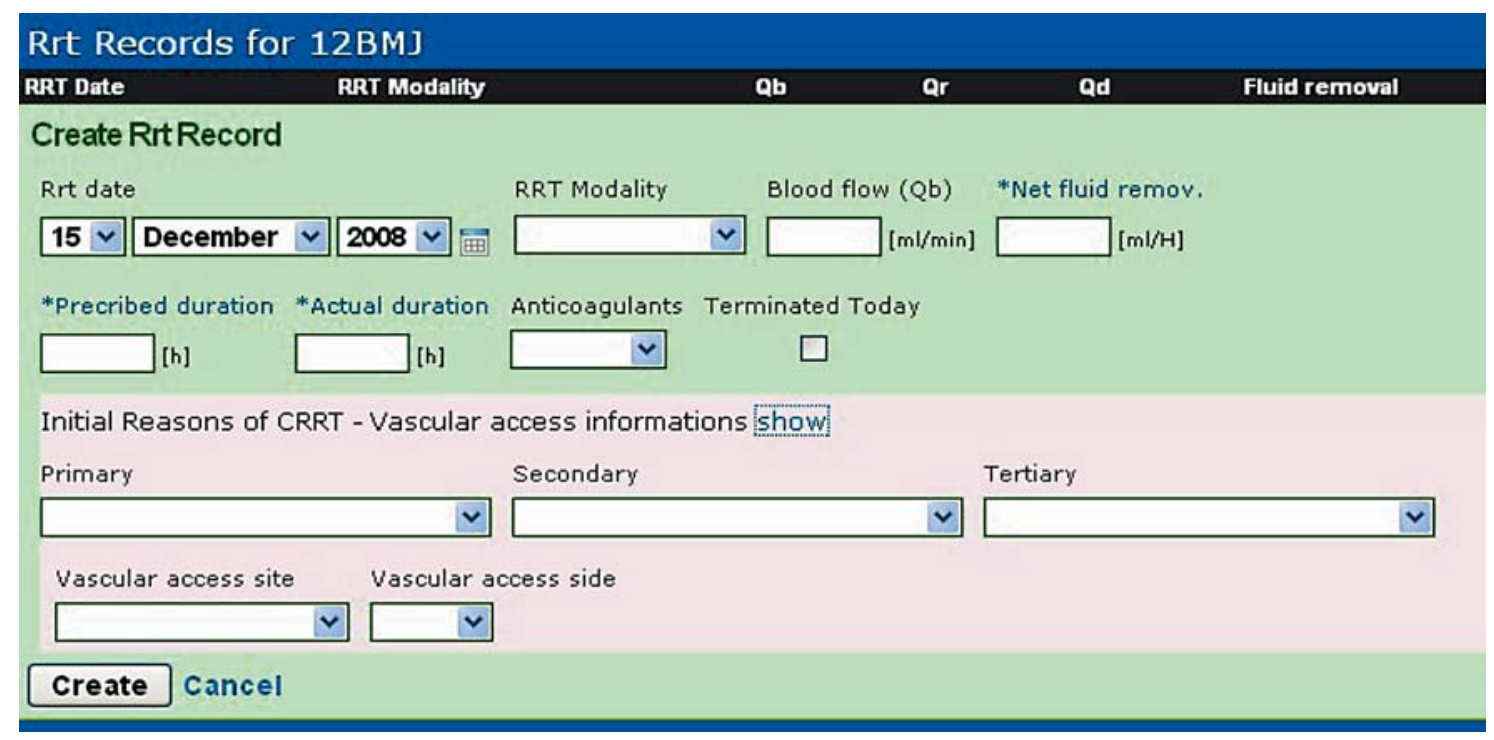

Fig. 11. The section is totally dedicated to CRRT treatment. Indication for RRT, details of RRT prescription and delivery are recorded here. 


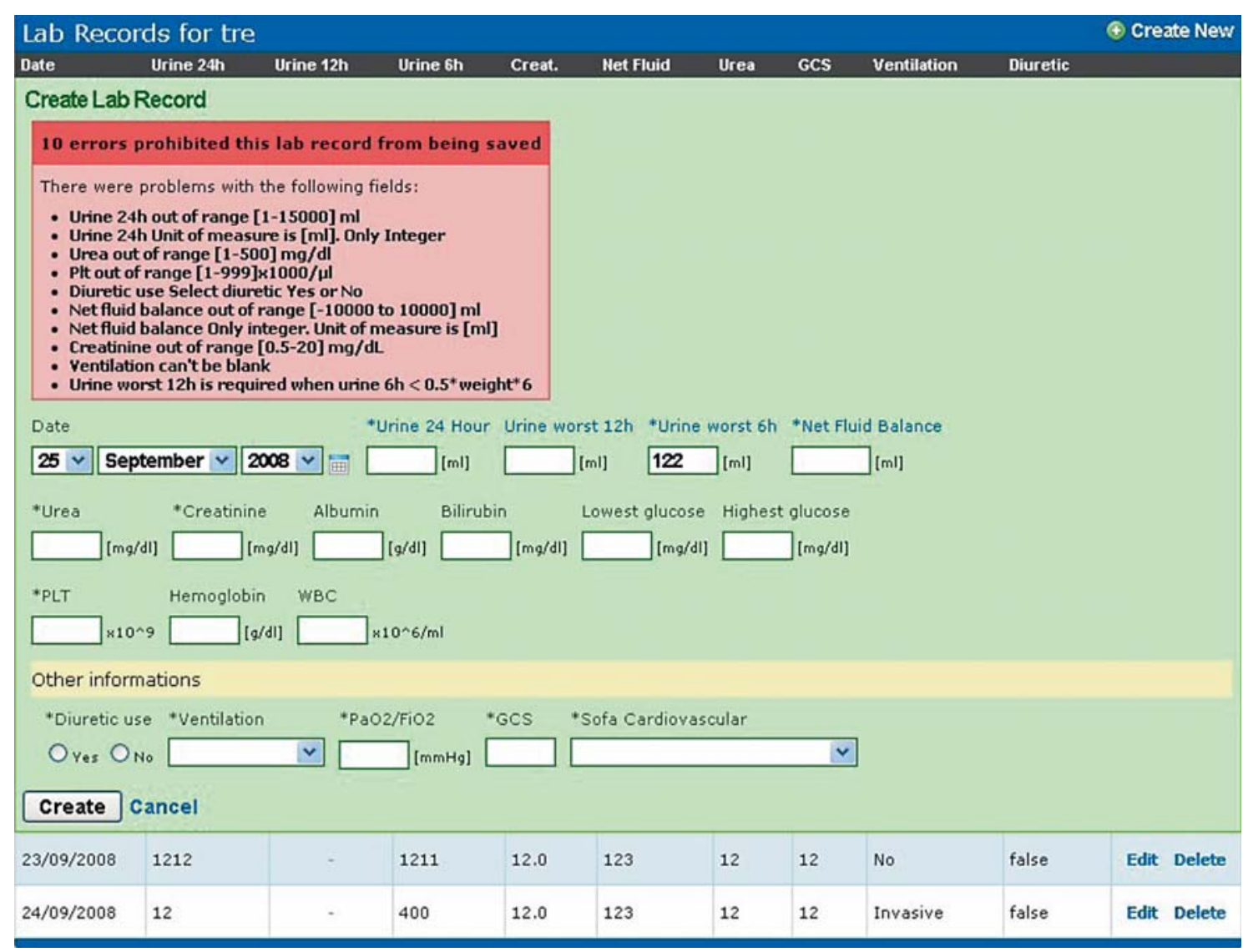

Fig. 12. Each section contains specific data verification tool. Missing, out of range, obligatory and 'conditioned' fields as well as inconsistencies in data are verified before the validation and registration of the section.

Among these patients, it took a median of 2 (IQR 1-6) days to attain their maximum RIFLE class. RIFLE-max was Risk in 127 patients (33.5\%), Injury in $130(34.3 \%)$ and Failure in 122 (32.2\%).

AKI patients were older, more likely to be male, and have a history of cardiovascular disease and hypertension, and to have had exposure to ACE-I or ARBs prior to their hospitalization. They were more likely to have a respiratory or trauma diagnosis on ICU admission, and had higher APACHE II, SAPS II, and SOFA scores. On their first ICU day, AKI patients were more likely to be on vasopressors, but the proportion of patients on mechanical ventilation was similar between the two groups. AKI patients also had lower mean arterial pressure, $\mathrm{UO}, \mathrm{pH}, \mathrm{pO}_{2}$ and $\mathrm{PaO}_{2} / \mathrm{FiO}_{2}$ values, and higher urea and $\mathrm{SCr}$ values on ICU admission. 116 AKI patients (30.6\%) fulfilled criteria for sepsis during their ICU stay, compared to $33(16.7 \%)$ of non-AKI patients $(\mathrm{p}<0.001)$.
48 patients (8.3\%) were treated with RRT in the ICU; 2/48 did not fulfill criteria for AKI and received RRT for nonrenal indications. Overall, patients were started on RRT a median of 2 (IQR 0-6) days after ICU admission. AKI patients were started on RRT a median of 1 (IQR $0-4)$ day after fulfilling criteria for AKI. Median duration of RRT was 5 (IQR 2-10) days.

AKI patients had a higher crude ICU mortality $(28.8$ vs. $8.1 \%$, non-AKI; $\mathrm{p}<0.001)$ and longer ICU length of stay (median 7 vs. 3 days, non-AKI; p < 0.001) (fig. 4). Crude ICU mortality and ICU length of stay increased with greater severity of AKI. 225 patients (59.4\% of AKI patients) had complete recovery of renal function, with an SCr at time of ICU discharge which was $\leq 120 \%$ of baseline; an additional 51 AKI patients (13.5\%) had partial renal recovery, while 103 (27.2\%) had not recovered renal function at the time of death or ICU discharge. 


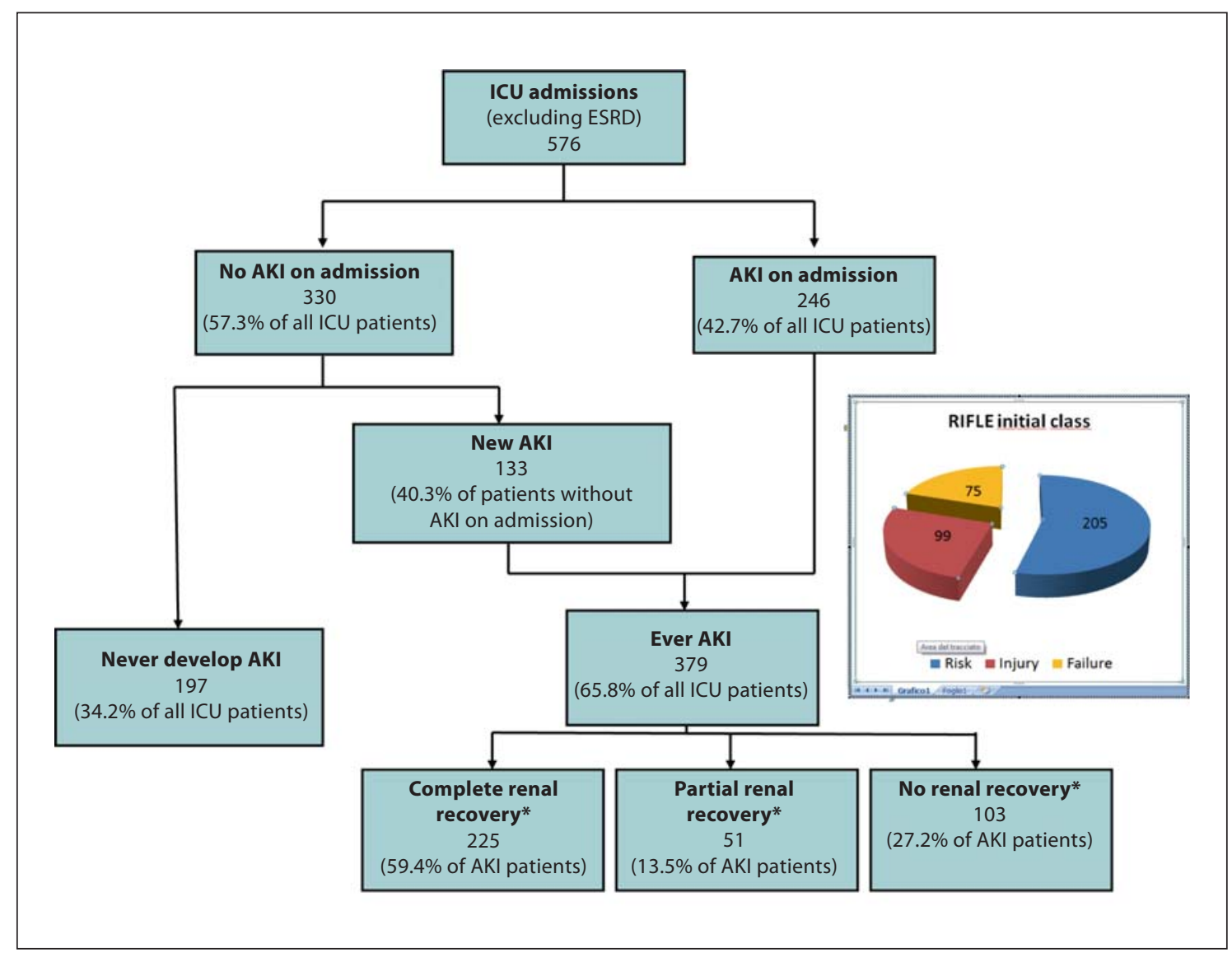

Fig. 13. Epidemiology of AKI derived from the NEFROINT database. The asterisk signifies at ICU discharge or death.

Sepsis events were recorded throughout patient's stay in the ICU; 149 patients (25.9\%) fulfilled criteria for sepsis. Of these, $116 / 149$ (77.8\%) had AKI, compared to 263/427 (61.6\%) nonseptic patients $(\mathrm{p}<0.001)$. Septic AKI patients tended to fulfill criteria for both sepsis and AKI on the same day; in a few cases, sepsis preceded AKI (median days from sepsis criteria to AKI criteria, 0, IQR 0-1). Septic patients had more severe AKI, and were more likely to receive RRT, had higher ICU mortality and longer ICU stay in agreement with international database results [11].

\section{Discussion}

This is the first Italian multicenter prospective data collection to establish the epidemiology of AKI in the ICU. The study confirms previous analyses describing
RIFLE as an optimal classification system to stage AKI severity. AKI is indeed a deadly complication for ICU patients where the level of severity correlated with mortality and length of stay. The tool developed for data collection was user friendly and easy to implement. Some of its features, including a RIFLE class alert system, may help the treating physician to collect systematically AKI data in the ICU and may possibly guide specific decision on the institution of RRT. Developing a user-friendly interface has been demanding and time-consuming. The approach was consistent with the current view that database entry methodology should be simple and easy to correct and control. Physicians and nurses in the ICU have very little time to comply with complicated studies requiring time-consuming procedures for data input. The current version of the NEFROINT database seems to respond to the requirements of simplicity together with internal accurate and sophisticated management of data. We feel 
Table 1. Characteristics of the cohort by presence or absence of AKI

\begin{tabular}{|c|c|c|c|c|}
\hline & All & AKI & Non-AKI & $\mathrm{p}$ \\
\hline Patients & 576 & 379 & 197 & \\
\hline Males & $352(59.4)$ & $237(62.5)$ & $105(53.3)$ & 0.039 \\
\hline Age, years & $66(53-76)$ & $69(58-78)$ & $59(43-72)$ & $<0.001$ \\
\hline Body weight, kg & $75(65-80)$ & $75(65-85)$ & $70(60-80)$ & $<0.001$ \\
\hline Height, cm & $170(160-175)$ & $170(160-175)$ & $170(162-175)$ & 0.274 \\
\hline Hypertension & $270(46.9)$ & $198(52.2)$ & $72(36.5)$ & 0.001 \\
\hline Diabetes & $108(18.7)$ & $79(20.8)$ & $29(14.7)$ & 0.2 \\
\hline Cardiovascular disease & $249(43.2)$ & $183(48.3)$ & $66(33.5)$ & 0.003 \\
\hline History of chronic kidney disease & $36(6.2)$ & $26(6.9)$ & $10(5.1)$ & 0.471 \\
\hline History of proteinuria or hematuria & $12(2.1)$ & $11(2.9)$ & $1(0.5)$ & 0.067 \\
\hline Baseline creatinine, mg/dl & $1.0(0.8-1.1)$ & $1.0(0.8-1.1)$ & $1.0(0.8-1.2)$ & 0.457 \\
\hline \multicolumn{5}{|l|}{ Medications } \\
\hline NSAID & $56(9.7)$ & $38(10.0)$ & $18(9.1)$ & 0.769 \\
\hline ACE-I or ARB & $95(16.5)$ & $71(18.7)$ & $24(12.2)$ & 0.045 \\
\hline \multicolumn{5}{|l|}{ Patient type } \\
\hline Medical & $273(47.4)$ & $194(51.2)$ & $79(40.1)$ & 0.014 \\
\hline Elective surgery & $117(20.3)$ & $73(19.3)$ & $44(22.3)$ & 0.385 \\
\hline Emergency surgery & $186(32.3)$ & $112(29.5)$ & $74(37.6)$ & 0.06 \\
\hline \multicolumn{5}{|l|}{ Admission diagnosis } \\
\hline Respiratory & $158(27.4)$ & $115(30.3)$ & $43(21.8)$ & 0.031 \\
\hline Neurologic & $98(17.0)$ & $50(13.2)$ & $48(24.4)$ & 0.001 \\
\hline Trauma & $83(14.4)$ & $44(11.6)$ & $39(19.8)$ & 0.012 \\
\hline Cardiovascular & $70(12.1)$ & $60(15.8)$ & $10(5.1)$ & $<0.001$ \\
\hline Gastrointestinal & $32(5.6)$ & $22(5.8)$ & $10(5.1)$ & 0.849 \\
\hline \multicolumn{5}{|l|}{ ICU admission } \\
\hline SOFA & $5(3-7)$ & $6(4-8)$ & $4(3-6)$ & $<0.001$ \\
\hline SAPS II & $43(35-54)$ & $44(37-55)$ & $42(32-53)$ & 0.0023 \\
\hline APACHE II & $18(13-24)$ & $19(14-25)$ & $17(12-21)$ & $<0.001$ \\
\hline Creatinine, $\mathrm{mg} / \mathrm{dl}$ & $0.9(0.7-1.4)$ & $1.1(0.8-1.6)$ & $0.8(0.6-1.0)$ & $<0.001$ \\
\hline Urea, mg/dl & $41(27-64)$ & $50(32-78)$ & $33(22-44)$ & $<0.001$ \\
\hline Urine output first ICU day, ml & $925(500-1,635)$ & $800(450-1,380)$ & $1,250(750-1,950)$ & $<0.001$ \\
\hline $\mathrm{Na}, \mathrm{mEq} / \mathrm{l}$ & $140(137-143)$ & $140(137-144)$ & $139(137-142)$ & 0.0232 \\
\hline $\mathrm{K}, \mathrm{mEq} / \mathrm{l}$ & $3.8(3.5-4.2)$ & $3.9(3.6-4.4)$ & $3.7(3.5-4.0)$ & $<0.001$ \\
\hline Bilirubin, mg/dl & $0.7(0.5-1.1)$ & $0.7(0.5-1.0)$ & $0.7(0.5-1.2)$ & 0.672 \\
\hline Hematocrit, \% & $32(28-36)$ & $32(28-36)$ & $32(28-35)$ & 0.913 \\
\hline $\mathrm{WBC}(\times 1,000)$ & $11(8-16)$ & $11(8-16)$ & $10(8-15)$ & 0.515 \\
\hline Platelets & $188(127-250)$ & $184(124-248)$ & $191(138-253)$ & 0.383 \\
\hline $\mathrm{pH}$ & $7.40(7.33-7.45)$ & $7.39(7.32-7.45)$ & $7.40(7.36-7.47)$ & $<0.001$ \\
\hline $\mathrm{pCO}_{2}, \mathrm{~mm} \mathrm{Hg}$ & $38(33-45)$ & $38(33-45)$ & $38(32-43)$ & 0.072 \\
\hline $\mathrm{pO}_{2}, \mathrm{~mm} \mathrm{Hg}$ & $114(82-156)$ & $110(79-150)$ & $122(88-161)$ & 0.026 \\
\hline $\mathrm{HCO}_{3}, \mathrm{mEq} / 1$ & $23(21-26)$ & $23(20-26)$ & $23(21-26)$ & 0.30 \\
\hline $\mathrm{PaO}_{2} / \mathrm{FiO}_{2}$ & $253(161-366)$ & $230(156-342)$ & $286(189-420)$ & $<0.001$ \\
\hline Mechanical ventilation, $\%$ & $532(92.4)$ & $353(93.1)$ & $179(90.9)$ & 0.326 \\
\hline Use of vasopressors, \% & $148(25.7)$ & $115(30.4)$ & $33(16.8)$ & $<0.001$ \\
\hline Dopamine $/$ dobutamine $\leq 5 \mu \mathrm{g} / \mathrm{kg} / \mathrm{min}$ & $45(7.8)$ & $37(9.8)$ & $8(4.1)$ & 0.014 \\
\hline \multicolumn{5}{|l|}{ Dopamine/dobutamine $\leq 5$ or } \\
\hline epinephrine/norepinephrine $\leq 0.1 \mu \mathrm{g} / \mathrm{kg} / \mathrm{min}$ & $45(7.8)$ & $31(8.2)$ & $14(7.1)$ & 0.744 \\
\hline Epinephrine $/$ norepinephrine $>0.1 \mu \mathrm{g} / \mathrm{kg} / \mathrm{min}$ & $58(10.1)$ & $47(12.4)$ & $11(5.6)$ & 0.009 \\
\hline Sepsis 1 & $149(25.9)$ & $116(30.6)$ & $33(16.7)$ & $<0.001$ \\
\hline \multicolumn{5}{|l|}{ Outcome } \\
\hline ICU mortality & $125(21.7)$ & $109(28.8)$ & $16(8.1)$ & $<0.001$ \\
\hline ICU length of stay, days & $5(3-14)$ & $7(3-16)$ & $3(2-8)$ & $<0.001$ \\
\hline
\end{tabular}

Figures in parentheses indicate percentage or range. 
that this approach may represent a potential pathway towards large multicenter studies to be undertaken in the near future.

\section{Participating Centers and Investigators}

Department of Anesthesiology and Intensive Care, St. Bortolo Hospital, Vicenza (Silvia Grammaticopolo, Marzia Dal Santo, Pasquale Piccinni). Department of Cardiocirculatory Physiopathology, Anesthesiology and Surgery, University of Rome 'La Sapienza', Rome (Monica Rocco, Elisa Alessandri). Division of Anesthesiology and Intensive Care, Department of Surgery, University of Pisa, Pisa (Francesco Giunta). Department of Intensive Care and Anesthesiology, Catholic University of Sacred Heart, Rome (Vincenzo Michetti). Intensive Care Unit, Department of Anesthesia and Resuscitation, Federico II University Hospital, Naples (Michele Iannuzzi). Intensive Care Unit, Emergency Department of Anesthesiological and Chirurgical Science 2, University Hospital, Naples (Clara Belluomo Anello). Department of An- esthesia and Intensive Care, San Giovanni di Dio Hospital, Florence (Giorgio Tulli). Anesthesia and Intensive Care Division, Emergency and Organ Transplantation Department, University of Bari, Bari (Nicola Brienza). Department of Anesthesia and Intensive Care, University of Verona, Azienda Ospedaliera Universitaria Integrata, Verona (Mauro Carlini). Intensive Care Unit, University Hospital, Ospedali Riuniti Umberto I, G.M. Lancisi, G. Salesi, Torrette (Paolo Pelaia, Vincenzo Gabbanelli). Department of Cardiothoracic Anesthesia and Intensive Care, Vita-Salute San Raffaele University, San Raffaele Scientific Institute, Milan (Tiziana Bove).

\section{Acknowledgement}

The study was supported by the national project Programma Strategico 'Costituzione di un network mutiregionale per la prevenzione della malattia renale e migliorare il management clinico del paziente nefropatico' of the Italian Ministry of Health (San Bortolo Hospital Institutional grant 222/2009-2010).

\section{References}

1 Bellomo R, et al: Acute renal failure - definition, outcome measures, animal models, fluid therapy and information technology needs: the Second International Consensus Conference of the Acute Dialysis Quality Initiative (ADQI) Group. Crit Care 2004; 8:R204-R212.

-2 Mehta RL, et al: Acute Kidney Injury Network: report of an initiative to improve outcomes in acute kidney injury. Crit Care 2007; 11:R31.

-3 Cruz DN, Ricci Z, Ronco C: Clinical review: RIFLE and AKIN - time for reappraisal. Crit Care 2009;13:211.

4 Ricci Z, Cruz D, Ronco C: The RIFLE criteria and mortality in acute kidney injury: a systematic review. Kidney Int 2008;73:538-546.

5 Ostermann M, Chang RW: Acute kidney injury in the intensive care unit according to RIFLE. Crit Care Med 2007;35:1837-1843.
-6 Bagshaw SM, et al: A multi-centre evaluation of the RIFLE criteria for early acute kidney injury in critically ill patients. Nephrol Dial Transplant 2008;23:1203-1210.

7 Thakar CV, et al: Incidence and outcomes of acute kidney injury in intensive care units: a Veterans Administration study. Crit Care Med 2009;37:2552-2558.

8 Cruz DN, et al: Diagnostic and staging criteria for acute kidney injury: do we need prospective studies? Minerva Anestesiol 2008; 74(suppl 1):303-305.

$\checkmark 9$ Fiaccadori E, et al: Predicting patient outcome from acute renal failure comparing three general severity of illness scoring systems. Kidney Int 2000;58:283-292.

10 Cruz DN, et al: North East Italian Prospective Hospital Renal Outcome Survey on Acute Kidney Injury (NEiPHROS-AKI): targeting the problem with the RIFLE Criteria. Clin J Am Soc Nephrol 2007;2:418-425.

-11 Uchino S, et al: Acute renal failure in critically ill patients: a multinational, multicenter study. JAMA 2005;294:813-818.
12 Uchino S, et al: An assessment of the RIFLE criteria for acute renal failure in hospitalized patients. Crit Care Med 2006;34:1913-1917.

$>13$ Levy MM, et al: 2001 SCCM/ESICM/ACCP/ ATS/SIS International Sepsis Definitions Conference. Crit Care Med 2003;31:12501256.

$>14$ Knaus WA, et al: APACHE II: a severity of disease classification system. Crit Care Med 1985;13:818-829.

15 Vincent JL, et al: Use of the SOFA score to assess the incidence of organ dysfunction/ failure in intensive care units: results of a multicenter, prospective study. Working group on 'sepsis-related problems' of the European Society of Intensive Care Medicine. Crit Care Med 1998;26:1793-1800.

16 Le Gall JR, Lemeshow S, Saulnier F: A new Simplified Acute Physiology Score (SAPS II) based on a European/North American multicenter study. JAMA 1993;270:2957-2963. 